\title{
Structure Changes Induced by the Milling of Crystalline Polymers
}

\author{
Norio KUROKAWA and Junkichi SOHMA \\ Faculty of Engineering, Hokkaido University, Sapporo 060, Japan.
}

(Received December 26, 1978)

\begin{abstract}
It was found through $\mathrm{X}$-rays diffraction pattern analyses that the crystalline structure of polypropylene changed from a monoclinic into a pseudohexagonal by milling. This crystal transformation was reversibly restored to the stable monoclinic structure by heat treatments of the fractured polypropylene. Decreases in both crystallite size and crystallinity were observed after the fracture and these decreases were found to be irreversible. The mobile fractions determined by the measurements of spin-spin relaxation times of the proton NMR were consistently interpreted with the conclusions determined by the X-ray analyses. Similar phenomena were also found on highdensity polyethylene.

KEY WORDS Polypropylene / Crystal Transformation / Milling / Fracture Crystallite Size / X-Rays / Heat Treatment / High-Density Polyethylene /
\end{abstract}

It has been established by ESR studies that when polymer chains are ruptured, they leave a couple of free radicals by the mechanical fracture of solid polymers at a low temperature. ${ }^{1-4} \mathrm{We}$ reported that the radicals formed by mechanical fracture (Mechano-Radicals) were trapped on the fresh surfaces produced by the ball-milling. ${ }^{5}$ It is known that mechanical fractures of inorganic materials affect the crystalline structures. ${ }^{6,7}$ However, the effect of the milling to crystalline structures have not been investigated in detailin case of polymeric materials. Thus, it is interesting to study the effects of the ballmilling to the crystalline structure of polymers. Polypropylene (PP) and high-density polyethylene (PE) were taken as examples on which the influence of the fracture was studied, since these are the simplest crystalline polymers and the information of their crystalline structures has accumulated.

\section{EXPERIMENTAL}

\section{Sample}

An isotactic polypropylene (PP, J400STD, $96 \mathrm{wt} \%$ isotactic) and high-density polyethylene (PE, Sholex 6050), manufactured by Mitsui Chemical Co. and Showa Denko Co., respectively, were used as samples after extraction of the antioxidants by use of hot toluene.

\section{Milling}

Milling was carried out by the ball-mill described in detail in a previous paper. ${ }^{8}$ Polymer flakes were placed in a glass ampoule which was then evacuated to $10^{-4}$ Torr $\left(\approx 133.322 \times 10^{-4} \mathrm{~Pa}\right)$. In this paper, the samples which have once undergone a mechanical fracture are called "fractured samples" and those orginal samples which have never been fractured are called the "nonfracture," for reference.

\section{$X$-ray Diffractometer}

$\mathrm{X}$-ray diffraction patterns of polypropylene and polyethylene were observed by using a Rigaku Denki $\mathrm{X}$-ray 2027 diffractometer $(\mathrm{Cu}-\mathrm{K} \alpha$, Ni:filtered $)$ at the room temperature.

\section{Pulse NMR and ESR Spectrometers}

Free induction decay (FID) experiments for determining spin-spin relaxation times, $T_{2}$ 's, were carried out by a SXP4-100 pulse NMR (Bruker) using $90 \mathrm{MHz}$. NMR sample tubes containing samples were evacuated to $10^{-4}$ Torr $\left(\approx 133.322 \times 10^{-4} \mathrm{~Pa}\right)$. Temperature variation experiments were carried out with a attachedtemperature control unit. ESR spectra were obtained by a PE-1 X band spectrometer (JEOL) with $100 \mathrm{KHz}$ modulation. 


\section{RESULTS}

\section{Changes in $X$-ray Profiles}

Polypropylene $(P P)$. Frakes of isotactic polypropylene $(\mathrm{PP})$ were milled in a vacuum of $10^{-4}$ Torr $\left(\approx 133.322 \times 10^{-4} \mathrm{~Pa}\right)$ at $77 \mathrm{~K}$ by the ball-mill. $\mathrm{X}$-ray diffraction profiles obtained before and after the milling are shown in Figure 1. Apparently, the profile (Figure 1a) before the fracture, which is known to have a monoclinic structure, ${ }^{9,10}$ changes drastically into a different one. X-ray diffraction patterns (Figure 1b) following the fracture were identified as pseudohexagonal, so-called smectic structures. These smectic structures were so unstable that the transition from the pseudohexagonal to the monoclinic form ( $\alpha$-modification) was induced by heat treatment at temperatures above $c a$. $343 \mathrm{~K}$. X-ray diffraction of the fracture PP after 24 hours' annealing at $385 \mathrm{~K}$, which is high enough for rapid transformation but lower than the recrystallization temperature, is shown in Figure 2. In this profile, the peaks corresponding to the monoclinic structure appear again, although the lines are broadened than before the fracture and the halo component from an amorphous part is considerably mixed. Increase of the halo in the profile was observed from the fractured sample even after the annealing. This indicates an irreversible increase of the noncrystalline phase in the fractured sample. Crystallinities were estimated by decomposition of the $\mathrm{X}$-ray profile as $71 \%$ and $53 \%$ for the nonfractured and the fractured and annealed PP, respectively.

Polyethylene (PE). X-ray diffraction profiles obtained before and after the milling of PE (Sholex 6050) are shown in Figure 3. Apparently the characteristic pattern of an orthorhombic structure before the fracture was drastically changed to the different profile in this case, too. Since a new peak at $19.3^{\circ}$, which appears clearly after the fracture, is a reflection of the (001) plane of the monoclinic, ${ }^{11}$ the mechanical fracture of polyethylene at $77 \mathrm{~K}$ causes a crystal transformation from the orthorhombic to the monoclinic. The profile after the fracture was superposed with the halo of an amorphous part. When the fractured sample was annealed at $353 \mathrm{~K}$ for 15 hours, the monoclinic lines almost disappeared, as shown in Figure 4. The crystalline transformation by the milling was observed not only at $77 \mathrm{~K}$ but also at room temperature. The crystallinities were estimated from the X-ray profiles as $77 \%$ and $59 \%$ for the

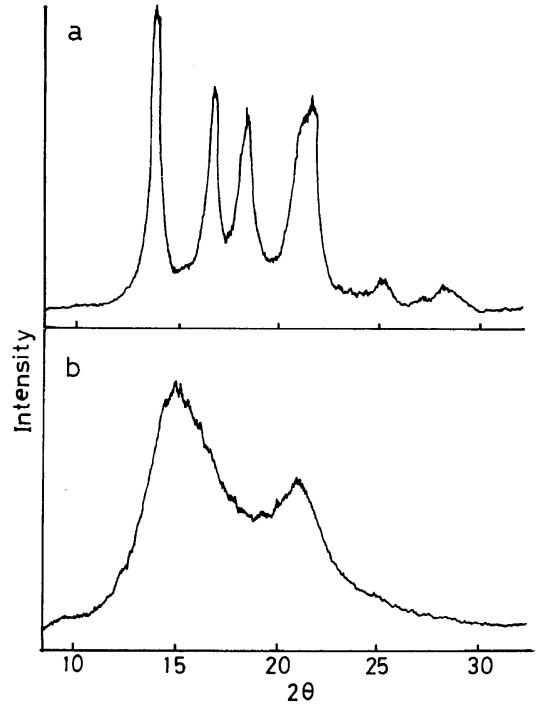

Figure 1. X-ray diffraction patterns of polypropylene: $\mathrm{a}$, before and $\mathrm{b}$, after milling at $77 \mathrm{~K}$ for 20 hours.

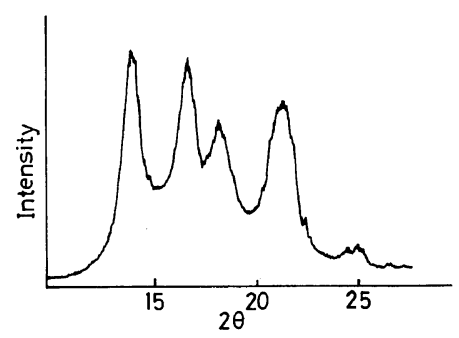

Figure 2. X-ray diffraction pattern of the fractured polypropylene after annelaing at $381 \mathrm{~K}$ for 24 hours.

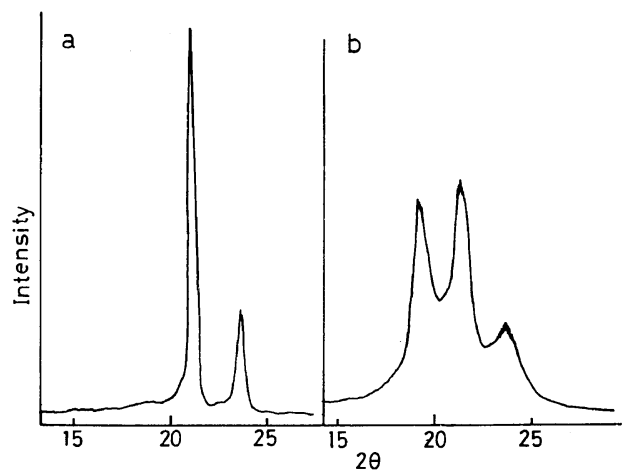

Figure 3. X-ray diffraction patterns of high-density polyethylene: $a$, before and $b$, after milling at $77 \mathrm{~K}$ for 20 hours. 
nonfractured and the fractured PE, respectively. Decreases in crystallinity with milling time were observed and are shown in Figure 5. No marked difference could be observed when the milling temperature was changed from $77 \mathrm{~K}$ to the room temperature. Comparing the profiles before and after the fracture, (Figure 3a and Figure 4), the decrease in crystallinity by milling is found to be irreversible, within the temperature range for the heat treatments in these experiments.

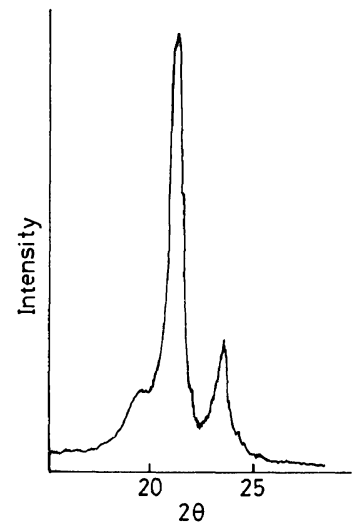

Figure 4. X-ray diffraction pattern of the fractured polyethylene annealing at $353 \mathrm{~K}$ for 15 hours.

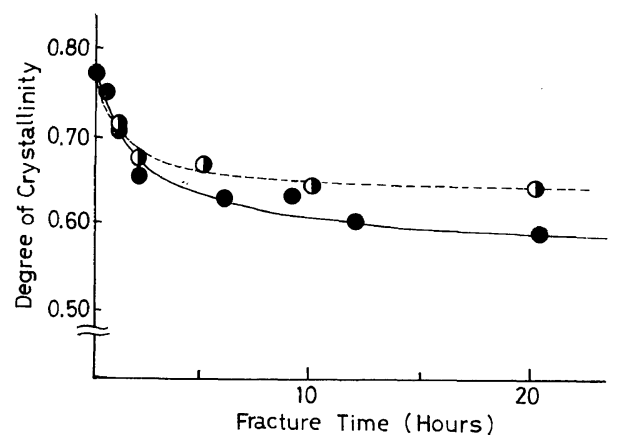

Figure 5. Changes in crystallinity of polyethylene with milling time: - milling at $77 \mathrm{~K} ; \mathbf{0}$, milling at $293 \mathrm{~K}$.

Conversion of the $\gamma$-Radicals in the Fractured Sample

A PP sample was ball-milled and the mechanoradicals in this milled sample were eradicated by storing a sufficiently long heat treatment at room temperature. After the complete annihilation of the mechano-radicals which had been checked by no ESR signal from the sample, this fractured PP was $\gamma$ - irradiated so as to produced radicals ( $\gamma$-radicals). Degassed methyl methacrylate (MMA) was introduced into an evacuated sample tube kept at $77 \mathrm{~K}$. The ESR spectrum observed from the sample is shown in Figure 6A. This spectrum is almost identical to that of the PP $\gamma$-radicals, reported in the literature. ${ }^{12,13}$ The spectrum from the same sample with MMA monomers after warming is shown in Figure 6B. Although the spectrum of " $B$ " in Figure 6 shows the characteristic nonet of PMMA propagating radical, ${ }^{14}$ the outermost peak of the PP $\gamma$ radicals, marked with the arrows, apparently survived. Similar experiments were performed for the nonfractured sample for comparison, but nonconversion of the $\gamma$-radicals was observed, as reported in the former paper. ${ }^{5}$ By decomposition of the observed spectrum " $B$ " into two components, the nonet of PMMA and the spectrum " $A$ " of the PP $\gamma$-radical, the conversion percentage was roughly estimated as $50 \%$.

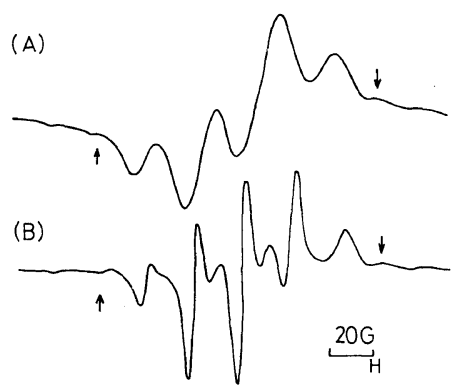

Figure 6. Comparison of ESR line shapes observed from PP $\gamma$-radicals in contact with MMA monomers (A) before and (B) after heat treatment; ESR observed at $77 \mathrm{~K}$.

\section{Differential Radial Distribution Function (DRDF) Analysis}

Differntial radial distribution function (DRDF) ${ }^{15}$ obtained by a Fourier transformation of the observed X-ray is shown in Figure 7. Characteristic behavior in DRDF of the fractured PP was not very different from that of the nonfractured one in a range less than $12 \AA$, but the periodicity in the DRDF of the fractured PP gradually decreased above $20 \AA$ and was finally lost in the long range. However, DRDF periodicity existed at a longer distance in the case of the nonfractured PP. 


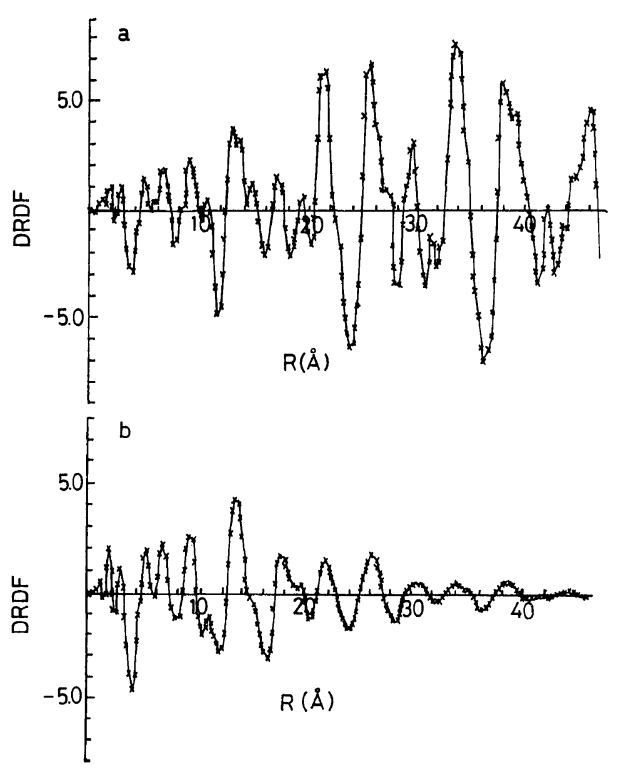

Figure 7. Differential radial distribution function of polyporpylene: $a$, the nonfractured $\mathrm{PP} ; \mathrm{b}$, the fractured PP.

\section{Mobile Fraction}

Spin-spin relaxation times, $T_{2}$ 's, were measured for PP samples by means of the pulse NMR. The logarithms of signal intensity of FID are plotted against time in Figure 8. In such a semi log plot, an experimental curve is decomposed into several components. ${ }^{16,17}$ The component having the shortest $T_{2}$ is linear not with time but with the square of time, as shown in the upper diagram in Figure 8. $T_{2}$ 's were estimated from the gradients of the linear plots of the components, named N, M, and B in the figure. The longer $T_{2}$, which corresponds to a narrower line width, is a contribution from the mobile component, and therefore a mobile fraction can be evaluated from the intercept of each component in Figure 8. The mobile fractions measured in such a way were determined at several temperatures and the temperature dependences of the mobile fractions are shown in Figure 9. The mobile fraction at $350 \mathrm{~K}$ apparently increases after the mechanical fracture. The temperature, at which the mobile fraction increases, is lower for the fractured samples. It is interesting to notice that the heat treatment of the fractured sample has little effect on the mobile fraction and the behavior of the annealed sample is close to the fractured one. This means that increase, due to fracture, in the

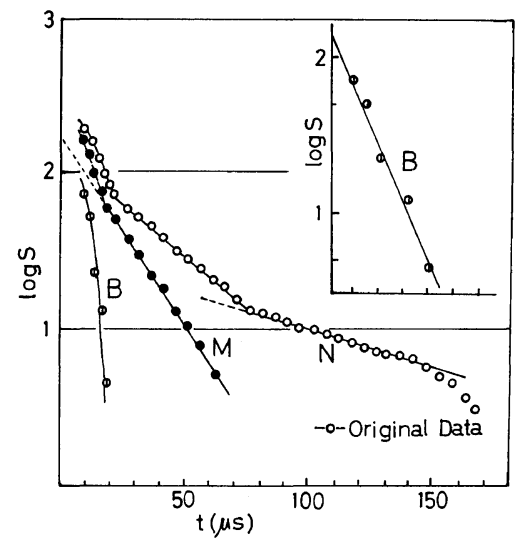

Figure 8. An example of the semilogarithm plot of the pulse heights $v s$. time; the fractured PP. Observed at $333 \mathrm{~K}$ with $90 \mathrm{MHz}$.

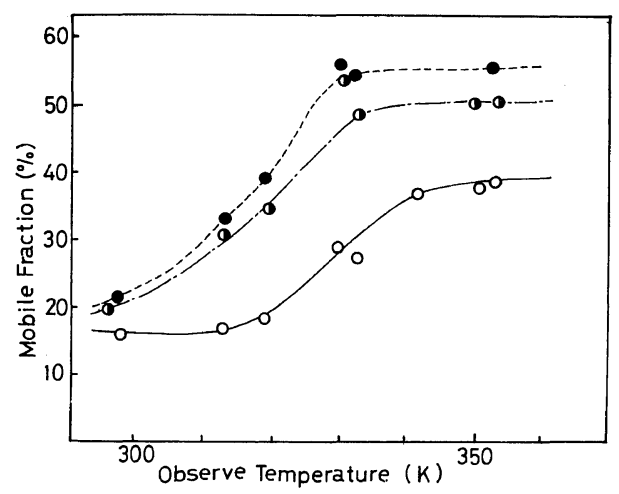

Figure 9. Temperature dependece of mobile fraction: $\mathrm{O}$, nonfractured PP; $\boldsymbol{O}$, fractured $\mathrm{PP}$ (at $77 \mathrm{~K}$ for 20 hours); , heat-treated PP after milling (at $381 \mathrm{~K}$ for 24 hours). NMR observed with $90 \mathrm{MHz}$.

mobile fraction that was hardly changed by heat treatment at $381 \mathrm{~K}$, was irreversible.

\section{DISCUSSION}

Crystal Transformation and Decrease in Crystallinity

It was found that the crystal transformations, from the monoclinic to the pseudohexagonal of PP and from the orthorhombic to the monoclinic structure of PE, were induced by mechanical fracture. On consideration of similar crystal transformation, which occurred by cold drawing or compression of the bulk samples, ${ }^{11,18}$ it is plausible that these crystal transformations are induced by impulses of the ball- 
mill. A similarity in effect on the crystalline structure suggests that strong stress is probably induced in the sample when milling is carried out.

Decreases of the crystallinity were found for both PP and PE after the mechanical fracture. These facts are consistent with the density measurement results of the polymers. The densities of PP were measured by the floating method using water-ethanol mixture, and the results are shown in Table I.

According to the literature ${ }^{18,19}$ the desnsities of the monoclinic, pseudohexagonal, and the amorphous phases of PP are $d_{\mathrm{c}}=0.936, d_{\mathrm{s}}=0.88$, $d_{\mathrm{a}}=0.85$, respectively. The crystallinity of the nonfractured sample is estimated as $78 \%$, using the observed density, $d_{\mathrm{c}}$ and $d_{\mathrm{a}}$. This value agrees well with that determined by the X-ray data. Similarily the crystallinity was estimated for the fracture and then the annealed sample as $66 \%$ which is apparently smaller than the nonfractured one. This irreversible decreases of crystallinity found by density measurements is consistent with the results by $\mathrm{X}$-ray analysis. The density of the fractured PP is a little larger than $d_{\mathrm{s}}$ and this difference is meaningful since error involved in the experiment is \pm 0.002 . This difference in observed density means that the fractured sample contains crystallites of higher density than $d_{\mathrm{s}}$, probably monoclinic. Therefore, the density measurements seem to suggest that the monoclinic structure survives following the fracture, although no apparent component of the monoclinic is observed in the X-ray profile (Figure $1 \mathrm{~b}$ ).

The increase in the mobile fraction was observed after the milling and was irreversible, as shown in Figure 9. Since the increase in the mobile fraction means a decrease in crystallinity, the irreversible increase of the mobile fraction after the fracture indicates an irreversible decrease in the crystallinity of the fractured samples.

The results obtained by these different experimental techniques, X-ray diffraction, density, and the mobile fraction measurements, consistently lead us to the conclusion that crystal transformations and the irreversible decrease in crystallinity were caused by the milling of the crystalline polymers, PP and PE.

\section{Decrease in Crystallite Size}

The line widths of the $\mathrm{X}$-ray diffraction peaks increase irreversibly in the fractured samples, as shown in Figures 2 and 4 . There are two possible origins for the line broadening in the $\mathrm{X}$-ray profile:
Table I. Comparisons of the crystallinity

\begin{tabular}{|c|c|c|c|c|}
\hline & & \multicolumn{2}{|c|}{ Crystallinity } & \multirow{2}{*}{ Density } \\
\hline & & $\begin{array}{l}\text { From } \\
\text { X-ray }\end{array}$ & $\begin{array}{c}\text { From } \\
\text { density }\end{array}$ & \\
\hline \multirow{3}{*}{\multicolumn{2}{|c|}{$\begin{array}{l}\text { PP Nonfractured } \\
\text { Fractured } \\
\text { Fractured and } \\
\text { Heat-treated }\end{array}$}} & $71 \%$ & $78 \%$ & 0.917 \\
\hline & & & & 0.897 \\
\hline & & $53 \%$ & $66 \%$ & 0.907 \\
\hline \multirow[t]{2}{*}{$\mathrm{PE}$} & Nonfractured & $77 \%$ & & \\
\hline & Fractured & $59 \%$ & & \\
\hline
\end{tabular}

decreased in crystallite size and increase in the disordering of the crystallites.

In the radical conversion experiments, described in the "Results", it was found that the $\gamma$-radicals in the fractured sample were scavenged considerably by bulky MMA monomers but no scavenging was observed in the case of the nonfractured sample. MMA monomers are too bulky to penetrate the PP crystallites, and a scavenging reaction by the MMA is reasonably assumed to occur on the surfaces of the crystallites. An increase in the number of scavenged $\gamma$-radicals means a relative increase in the surface to volume ratio of the crystallites, provided that $\gamma$ irraiation produces radicals uniformly throughout the sample. This increase in the surface to volume ratio arises from the decrease in crystallite size. Accordingly, the experimental results on the radical conversion suggest strongly that the decrease in crystallite size was caused by the milling of the sample. Less ordering in the long range in the milled sample, as was concluded from the DRDF experiments, is understood as a decrease in size of the crystallite.

Based on this conclusion, the decrease in crystallite size is reasonably considered as the origin of line broadening in the X-ray profile after the fracture. So, Scherrer's equation, ${ }^{20}$

$$
t_{h k l}=\frac{k \lambda}{\beta \cos \theta}
$$

is now used for estimating the crystallite size from the line width of the X-ray diffraction pattern. In this expression, $t_{h k l}$ stands for the size of the $(h k l)$ plane, $\lambda$ for the wavelength of used X-ray, $\beta$ for the integral breadth, $\theta$ for the peak position, and $k$ is a constant taken as 1.05 . By using this equation the size of the 
(110) plane was calculated from the observed breadth. The calculated crystallite sizes are plotted as a function of milling time in Figure 10. The crystallite size decreases rapidly in a short time but becomes constant after approximately 5 hours. No marked dependence on the temperature of the fracture was observed. It is noteworthy that the decrease in crystallite size brought on by milling was also irreversible, just as the crystallinity was.

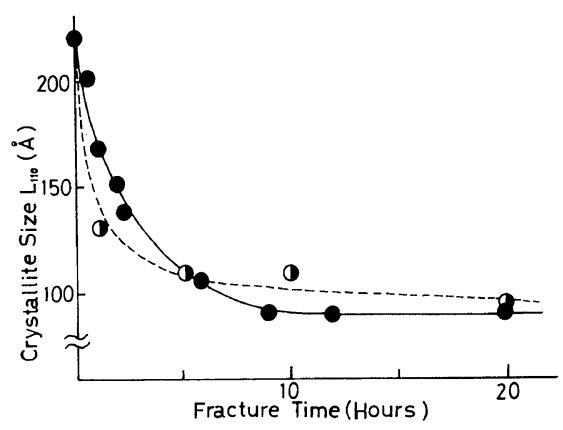

Figure 10. Milling time dependence of crystallite size in high-density polyethylene (PE): $\mathbf{O}$, milling at $77 \mathrm{~K}$, milling at $293 \mathrm{~K}$.

The crystallite size can be estimated from the observed line widths of higher-order diffraction of Xrays, using the following equation ${ }^{20,21}$

$$
\beta^{2}=\frac{1}{L_{h k l}^{2}}+\frac{(\pi g)^{4} m^{4}}{d_{h k l}^{2}}
$$

where the crystallite size and degree of distortion in the crystallite are represented by $L$ and $g$, and $m$ and $d_{h k l}$ are the reflection order and distance of the $(h k l)$ plane, respectively. Both the size and degree of distortion are determined from the gradient and the intercept of the Hosemann plot, ${ }^{21}$ although such a plot always involves rather larger error due to the poor $\mathrm{S} / \mathrm{N}$ of the higher-order diffraction line and the limited data points. These analyses have shown that sizes decrease from $120 \AA$ to $76 \AA$ and from $220 \AA$ to $92 \AA$ for PP and PE, respectively, and that no marked changes in the distortion occur after the milling. Bearing in mind the errors involved, the results are meaningful. The results mentioned above, the radical conversion, the analyses by Scherrer's equation and Hosemann plot consistently point to the conclusion that the irreversible decrease in the crystallites was induced hy milling.
Acknowledgement. The authors should like to express their gratitude to Prof. A. Odajima and Mr. T. Ishibashi in the Department of Applied Physics, Faculty of Engineering, Hokkaido University, for their kind assistance and valuable discussion on Xray analysis experiments. The authors are also grateful to the Coal Research Institute and NMR Laboratory of the Faculty of Engineering, Hokkaido University for the kind permission granted in using the apparatus there.

\section{REFERENCES}

1. T. Kawashima, S. Shimada, H. Kashiwabara, and J. Sohma, Polym. J., 5, 135 (1973).

2. M. Sakaguchi, H. Yamakawa, and J. Sohma, J. Polym. Sci., Polym. Lett. Ed., 12, 193 (1974).

3. M. Sakaguchi and J. Sohma, Polym. J., 7, 490 (1975).

4. J. Sohma and M. Sakaguchi, Adv. Polym. Sci., 20, 109 (1976).

5. N. Kurokawa, M. Sakaguchi, and J. Sohma, Polym. $J ., 10,93$ (1978)

6. K. Kubo in "Mekano Kemisutori Gairon (Introduction of Mechano Chemistry)," 2nd ed, Tokyo Kagaku Dojin Publing Co., Tokyo, 1978, Chapter 5.

7. Y. Sadahiro and K. Shimizu, J. Soc. Mater. Sci. Jpn., 18, 471 (1969).

8. M. Sakaguchi and J. Sohma, J. Polym. Sci. Polym. Phys. Ed., 13, 1233 (1975).

9. G. Natta, Makromol. Chem., 35, 94 (1959).

10. A. Turner-Jones, J. M. Aizleweel, and D. R. Beckett, Makromol. Chem., 75, 134 (1964).

11. T. Seto, T. Hara, and K. Tanaka, Jpn. J. Appl. Phys., 7, 31 (1968).

12. H. Yoshida and B. Bånby, Acta Chem. Scand., 19, 72 (1965).

13. S. Nara, H. Kashiwabara, and J. Sohma, J. Polym. Sci., A-2, 5, 929 (1967).

14. R. J. Abraham, H. K. Melville, D. H. Ovenall, and D. H. Whiften, Trans. Faraday Soc., 54, 1133 (1958).

15. I. Voigt-Margin, and F. C. Mijlhoff, J. Appl. Phys., 46, 1165 (1975).

16. K. Fujimoto, T. Nishi, and R. Kado, Polym. J., 3, 448 (1972).

17. T. Yokono and Y. Sanada, Fuel, 57, 334 (1978).

18. T. Takagi and H. Sasaki in "Poripuropiren Jushi (Polypropylene Resin)," 2nd ed, Nikkan Kogyo Shinbunsha Co. Ltd., Tokyo, 1970, Chapter 3.

19. G. W. Schael, J. Appl. Polym. Sci., 10, 901 (1966).

20. Leroy E. Alexander, "X-ray Diffraction Methods in Polymer Science," John Wiley \& Sons, Inc., New York, N.Y., 1969, Chapter 7.

21. R. Bonart, R. Hosemann, and R. J. McCullough, Polymer, 4, 199 (1963). 